Humanités numériques

\title{
À la recherche des haïdouks. Annoter les entités spatiales dans des romans roumains du XIX ${ }^{\mathrm{e}}$ siècle
}

Looking for Hajduks. Space Entities Annotation in Nineteenth-Century Romanian Novels

Ioana Galleron, Roxana Patraș, Camelia Grădinaru et Frédérique MélanieBecquet

\section{OpenEdition}

\section{Journals}

Édition électronique

URL : https://journals.openedition.org/revuehn/1399

DOI : 10.4000/revuehn.1399

ISSN : 2736-2337

Éditeur

Humanistica

\section{Référence électronique}

Ioana Galleron, Roxana Patraș, Camelia Grădinaru et Frédérique Mélanie-Becquet, « À la recherche des haïdouks. Annoter les entités spatiales dans des romans roumains du xix siècle », Humanités

numériques [En ligne], 3 | 2021, mis en ligne le 01 mai 2021, consulté le 15 juillet 2021. URL : http:// journals.openedition.org/revuehn/1399; DOI : https://doi.org/10.4000/revuehn.1399

Les contenus de la revue Humanités numériques sont mis à disposition selon les termes de la Licence Creative Commons Attribution 4.0 International. 


\title{
humanités numériques
}

$3 \mid 2021$

Humanités numériques spatialisées

INFORMATION SPATIALE ET CORPUS TEXTUELS

\section{À la recherche des haïdouks. Annoter les entités spatiales dans des romans roumains du $\operatorname{XIX}^{\mathbf{e}}$ siècle \\ Looking for Hajduks. Space Entities Annotation in Nineteenth-Century Romanian Novels}

\author{
Ioana Galleron, Roxana Patraș, Camelia Grădinaru et \\ Frédérique Mélanie-Becquet
}

\section{Résumés}

L'article présente une campagne d'annotation des entités spatiales dans un corpus de romans roumains du $\mathrm{XIX}^{\mathrm{e}}$ siècle ayant comme personnage principal le haïdouk, hors-la-loi des Balkans. En raison de leur mode de vie nomade, ces brigands ont une relation à l'espace qui se construit en opposition à l'organisation stable et structurée de l'espace rural roumain de l'époque. Pour vérifier si cette différence se retrouve dans les romans, l'objectif de l'annotation a été de relever tous les syntagmes nominaux et les adverbes faisant référence à l'espace, sans se cantonner aux toponymes, qui font habituellement l'objet des travaux en humanités numériques et en traitement automatique des langues. Après une présentation des principales différences entre l'approche adoptée et les travaux actuels ou plus anciens en analyse de l'espace littéraire, nous présentons ici les étapes d'élaboration du schéma d'annotation, les résultats et les enseignements de la mesure de l'accord interannotateurs sur dix échantillons d'environ mille mots chacun.

This paper presents the annotation of spatial entities in a corpus of Romanian novels written mainly in the 19th century and featuring as their main character a figure very well-known in the Balkans, the Hajduk outlaw. Because of their nomadic lifestyle, Hajduks were experiencing a unique relation to space, which was very different from that of the majority of Romanians, who lived in villages at the time. To verify if this 
different vision reverberates in novels, the aim was to collect all nominal and adverbial phrases referring to space, thus going largely beyond toponyms, that are usually marked-up and examined in digital humanities and natural language processing projects. After a presentation of the main differences between our approach and those prevailing in older or more recent studies in spatial literary analyses, we focus on the elaboration of the annotation scheme and comment upon the results of the inter-annotator agreement, as measured on a set of ten fully-annotated samples, of a thousand words each, from our novels.

\section{Entrées d'index}

MOTS-CLÉS : humanités numériques spatialisées, annotation, corpus d'auteur, littérature, roman, entités nommées

KEYWORDS: annotation, spatial digital humanities, authorial corpus, literature, novel, named entities

Ce travail a été financé par le ministère roumain de la Recherche et de l'Innovation (CCCDI-UEFISCDI, numéro PN-III-P3-3.1-PM-RO-FR-2019-0063/13 BM-2019, dans PNCDI III).

Un des personnages les plus fascinants de la littérature balkanique est celle du haïdouk - littéralement « hors la loi » en turc ${ }^{1}$-, un brigand dont la présence est attestée $d u x{ }^{\mathrm{e}}$ au début $d u x^{\mathrm{e}}$ siècle, et dont la figure pittoresque et inquiétante a fait l'objet d'une riche élaboration imaginaire. Pour s'en tenir seulement à l'espace roumain, dont il sera question dans cet article, on constate une présence constante et abondante de ce personnage entre 1860 et 1930 dans une large variété de formes artistiques : mélodrames, romans, nouvelles, pièces de théâtre, livrets, scénarios de film, etc. Sa prévalence est telle qu'entre 1840 et 1920 , approximativement $12 \%$ du nombre total de titres et résumés indexés dans le Dictionnaire chronologique du roman roumain (Dicționarul cronologic al romanului românesc 2003) fait référence à des haïdouks.

Depuis longtemps, la critique littéraire a observé que le succès de cette figure est lié à la fois à l'imaginaire qu'elle sollicite, et à son efficacité narrative. En effet, doté de qualités de courage, d'astuce et de débrouillardise, le personnage devient un héros populaire face aux représentants de l'ordre, souvent iniques ${ }^{2}$, et un symbole de résistance nationale devant l'oppresseur ottoman et le profiteur grec ${ }^{3}$. Par ailleurs, partageant un même éthos d'" honneur et de honte " avec des hors-la-loi occidentaux, on peut identifier à son sujet la même "tension entre le statut légal du personnage (proscrit, mauvais) et sa caractérisation actantielle qui a donné lieu à l'un des plus puissants et répandus motifs de l'imaginaire littéraire européen " (Leersen 2010, 407).

Plus rarement a-t-on observé que le haïdouk peut être également vu comme un vecteur de modernisation, en raison de sa vie nomade porteuse de valeurs comme la liberté individuelle, la démocratisation de l'accès à des produits de luxe et des commodités ou la mobilité des classes sociales. Rappelant à l'envi son association à la forêt et à la montagne, la critique en fait plutôt une sorte de sauvage, dont l'existence re- 
poserait sur une forme consentie d'involution par rapport à l'état donné d'une civilisation. Se pose toutefois la question des espaces associés aux haïdouks dans les romans : sont-ils toujours aussi désolés et écartés qu'on le suggère, ou bien des ancrages plus divers et plus diversement significatifs peuvent-ils y être identifiés?

Répondre à une telle question - et plus largement à celle de la nature et de la récurrence des espaces romanesques -, fût-ce dans un corpus limité et typé comme celui que nous avons en vue, n'est toutefois une opération facile. Car, si la notion de " chronotope ${ }^{4}$ " est ancienne dans la narratologie, et que plusieurs études sur l'espace romanesque se sont succédé, les méthodes pour l'identification de ceux-ci dans les textes littéraires sont rarement détaillées. Par ailleurs, la problématique posée plus haut demande d'analyser non pas un petit nombre d'occurrences jugées significatives en fonction du flair de l'interprétant, mais l'ensemble des entités spatiales présentes dans les textes. En se tournant du côté des outils numériques, réputés capables de réaliser de tels relevés complets, on constate toutefois que beaucoup de temps et d'énergie ont été dépensés pour l'identification des toponymes ${ }^{5}$, en tant que sous-classe des entités nommées, mais qu'une approche globale de l'expression de l'espace, comme celle dont nous aurions besoin pour étudier nos romans à haïdouks, reste à venir. En effet, le workshop sur les "geohumanities ", ainsi que la session "Theorising Spatial Humanities » de DH 2019 (Utrecht), ont donné des exemples très inspirants ${ }^{6}$ de visualisation géolocalisée d'œuvres littéraires, prenant le relais de toute une série d'approches qui mettent en dialogue parole écrite et cartes ${ }^{7}$. Cependant, malgré leur indéniable intérêt, ces études sont peu opérationnelles dans notre perspective peu focalisée sur des éléments géolocalisables, et encore moins sur le dialogue entre système d'information géographique (SIG) et textes.

L'élaboration d'une méthode plus globale d'identification et de caractérisation des entités spatiales (ES), dans un sens large, est donc nécessaire. Or, qu'elle tire parti d'une annotation manuelle ${ }^{8}$, d'un système associant lexiques et grammaires ${ }^{9}$, ou d'une forme d'intelligence artificielle $^{10}$, une telle méthode pose comme préalable l'existence d'une conceptualisation suffisamment stable de l'espace littéraire pour qu'elle puisse être appliquée par différents annotateurs - et, éventuellement et ultérieurement, par des machines - avec un taux satisfaisant de concordance. En d'autres mots, une étude à grande échelle, voire exhaustive, de l'espace associé aux haïdouks nous impose de réfléchir d'abord à un schéma d'annotation, et d'en tester la robustesse en vue d'une éventuelle production "de masse ${ }^{11}$ ". Mettant pour l'instant entre parenthèses l'analyse des haïdouks comme vecteurs de modernisation, et même, plus modestement, l'établissement d'une carte du territoire des haïdouks, tel qu'inféré des romans ${ }^{12}$, c'est à ces questionnements méthodologiques au croisement de la théorie littéraire, du traitement automatique des langues et de l'édition numérique que tentera de répondre cet article.

Après une brève présentation de notre corpus, nous montrerons comment, dans un va-et-vient entre différentes théorisations de l'espace mais aussi une pratique manuelle d'encodage, nous avons élaboré progressivement une liste de valeurs adaptées à l'analyse du texte littéraire. Par la suite, en nous appuyant sur les résultats d'une mesure de l'accord interannotateurs ( $\left.\mathrm{AIA}^{13}\right)$, dont nous exposerons la méthodologie, nous 
tâcherons de répondre à la question plus globale de l'automatisation de l'identification des entités spatiales. Loin de constituer un enjeu uniquement dans la perspective du traitement automatique des langues, cette interrogation a un impact direct sur l'analyse littéraire, dans la mesure où une réponse négative impliquerait que la construction de l'espace dans un roman relève avant tout d'effets de lecture, imposant, de ce fait, une interprétation plus tournée vers la subjectivité des récepteurs que vers un «donné » du texte.

\section{Présentation du corpus}

L'expérience d'annotation des entités spatiales dont il sera question dans les pages qui suivent s'ancre dans une campagne plus vaste de numérisation de romans roumains, principalement $\mathrm{du} \mathrm{XIX}^{\mathrm{e}}$ siècle. Cette campagne, commencée par le Digital Humanities Laboratory de l'université Alexandru Ioan Cuza de Iași, s'inscrit d'une part, dans le cadre de l'action COST 16204 Distant Reading ${ }^{14}$, et d'autre part, dans le cadre d'un projet franco-roumain Brâncuși intitulé $H A I-R O^{15}$.

De la totalité des romans à haïdouks identifiés par le Dictionnaire $d u$ roman roumain mentionné plus haut, HAI-RO s'est focalisé sur un sousensemble formé de quelque 50 titres, écrits entre 1850 et 1950 par des auteurs comme N.D. Popescu, G. Baronzi, Th. Stoenescu, P. Popescu, P. Macri, A. Marcu, Bucura Dumbravă, P. Istrati, C. Mătasă et quelques autres (voir Annexe). À noter que, à l'exception de cinq romans de Panait Istrati, Radu Rosetti et Bucura Dumbravă, écrits pour un lectorat éduqué et international (quatre de ces œuvres étant initialement rédigées en français ou en allemand), tous ces textes sont destinés à un public populaire, comme le montrent la basse qualité du papier et les inconsistances typographiques que l'on y constate, le recours fréquent à l'anonymat, et enfin l'absence de certaines informations éditoriales, comme (le plus compliqué dans notre cas) la date de publication, qu'il a fallu inférer de toute une série d'indices matériels et contextuels.

Les romans retenus ont été tout d'abord numérisés en mode image, puis ont fait l'objet d'une transcription grâce à l'entraînement d'un modèle d'OCR sous Transkribus. Dans une troisième phase, nous avons procédé au nettoyage manuel des sorties d'OCR et à l'annotation structurelle (parties, chapitres, paragraphes, différentes autres balises structurelles) en XML-TEI. Les résultats de cette chaîne de traitement, presque entièrement achevée à la date de rédaction de cet article, ont vocation à nourrir en partie la bibliothèque numérique du roman roumain et, en partie, la collection roumaine d'ELTeC (Patraș, Burnard et Odebrecht 2019 ${ }^{16}$ ).

\section{L'élaboration du schéma d'annotation}

Comment annoter les entités spatiales dans un roman ? La réponse à cette question implique de donner des consignes claires permettant d'une part, leur identification non ambiguë, et d'autre part, leur classement.

D'un point de vue linguistique, les entités spatiales sont construites grâce à une grande variété de lexèmes locatifs (toponymes, noms communs de lieux, syntagmes nominaux combinant les deux ${ }^{17}$ ), et d'élé- 
ments morphosyntaxiques (adpositions, affixes, flexions ${ }^{18}$ ), créateurs de points de repère, non seulement à partir d'objets larges et immobiles, mais aussi à partir d'objets petits et fréquents, ou dotés d'une signification culturelle dans une société partageant une histoire commune (Svorou 1994). Relever les entités spatiales signifie ainsi, concrètement, se focaliser sur les noms et les adverbes porteurs d'un sémantisme spatial, en prenant en compte l'ensemble du syntagme nominal, pronominal ou adverbial, c'est-à-dire avec les prépositions, les déterminants et les expansions diverses qu'il peut recevoir.

En ce qui concerne la caractérisation des ES, une conceptualisation de l'expression de l'espace dans la perspective de la mise en place d'une norme ISO a été entreprise par Pustejovsky et Lee $\left(2017^{19}\right)$. Les points spatiaux y sont conçus comme une catégorie de base à côté de cinq autres (objets, points temporels, événements, mesures, valeurs de vérité). Un ou plusieurs points spatiaux, mais aussi des objets, forment des entités spatiales, qui sont de trois genres : un lieu ("place»), un chemin (" path») et une trajectoire (" trajectory»). Pustejovsky et Lee recommandent également l'annotation des mesures et des relations spatiales, qui peuvent être qualitatives, d'orientation, de mouvement ou métriques.

Si intéressante que soit cette proposition pragmatique, son application à un objet littéraire ne va pas de soi, puisque « les concepts d'espace et de place employés par les humanistes ${ }^{20}$ sont fréquemment métaphoriques et non pas géographiques ». Selon les mêmes auteurs, "[les] récits [littéraires] se focalisent non pas sur l'espace, qui est un concept géométrique abstrait, mais plutôt sur les lieux, qui sont une expression particulière de l'espace géographique " (Bodenhamer, Corrigan et Harris 2015, 14) ; même si une telle focalisation reste discutable et devrait être démontrée au moyen d'explorations étendues de corpus, comme celle dont nous cherchons à poser ici les bases, la citation traduit un sentiment prévalent parmi les spécialistes de la littérature quant à la différence entre la façon dont l'espace s'actualise dans les discours non littéraires, et son utilisation dans des textes portés par une intention esthétique ${ }^{21}$. Cette différence, la géocritique l'explique, plus récemment, par la fréquence de la focalisation égocentrée dans l'espace littéraire ${ }^{22}$, autre particularité dont le schéma de Pustejovsky ne permet pas vraiment de rendre compte. Par ailleurs, une des questions principales dans les études littéraires est celle de la référence : «l'un des plus grands débats concerne l'inscription du texte dans l'espace, ou de l'espace dans le texte : la littérature est-elle connectée au réel par l'espace qu'elle représente ? Dans sa dimension spatiale, est-ce qu'on peut dire que le texte de fiction a un référent dans le soi-disant monde réel ?» (Westphal 2007)

Sur ce sujet, plusieurs conceptualisations existent. Ainsi, dans une perspective linguistique, rejointe sur ce point par la philosophie et la neurologie, la référence peut être " relative ${ }^{23}$ ", " absolue » ou " pragmatique ». De son côté, la psychologie développementale et behavioriste distingue plutôt entre références " égocentrées " et " allocentrées "; la théorie de la vision et de l'imaginaire oppose référence " centrée sur le regard " et référence " centrée sur l'objet "; les théories de la perception visuelle parlent de références " orientatives » vs. références « non orientatives "; tandis que la psycholinguistique parle de « vision centrée sur le récepteur ", de « vision centrée sur l'objet » et de « vision centrée sur l'en- 
vironnement » (Levinson 2014, 26). Comme on peut le constater, toutes ces théories présentent d'indéniables proximités, tout en n'étant que partiellement superposables. Elles suggèrent, toutes, des points d'attention et des valeurs que l'on peut souhaiter attacher aux entités spatiales identifiées dans une œuvre littéraire.

En tenant compte de ces suggestions, des besoins de l'analyse littéraire qu'elles mettent en avant et de la nécessité de proposer un schéma relativement facile à implémenter, nous avons opté pour la création d'une liste "à plat ", complétée d'un ensemble d'attributs permettant de capter des aspects plus complexes. Initialement, notre liste comportait six catégories :

- Les toponymes, définis comme des entités spatiales nommées, et qui peuvent être trouvés sur une carte. Si Pustejovsky et Lee les inscrivent parmi les « lieux », leur identification séparée semble essentielle dans la perspective de la référence, même si l'on considère que leur valeur est plus métaphorique que géographique.

- Les lieux, considérés comme des "toponymes non nommés », si l’on peut dire, ou comme des "éléments entopiques ": lexèmes du relief, noms communs désignant les différents types de localités, les bâtiments et les parties de bâtiment, les corps célestes, et ainsi de suite. Même si l'écrivain ne leur donne pas de nom (un village, la maison, etc.), ils en ont probablement un ou pourraient en avoir un.

- Des zones : espaces en principe non nommés et non nommables, définis nécessairement en relation avec un objet, un toponyme, un personnage ou une autre entité spatiale ${ }^{24}$. Il s'agit d'adverbes comme "dehors » ou d'expressions à pivot nominal ou pronominal, comme "à côté de la forêt ", "au-dessus du village ", " parmi eux ", "les environs de Bucarest ", etc.

- Les chemins, qui correspondent aux «paths » du schéma de Pustejovsky. Moins clairement conceptualisés dans l'analyse littéraire, les " chemins " peuvent difficilement être assimilés aux lieux ou aux zones.

- Les véhicules, qui sont des «lieux» d'un type particulier.

- Les adresses.

Comme indiqué plus haut, quelques attributs pouvaient être ajoutés à ces étiquettes, afin d'en augmenter l'expressivité. Tout particulièrement, l'annotation des zones nécessite de pouvoir pointer vers l'objet, le toponyme ou l'entité spatiale par rapport auxquels elles sont définies, et qui permettent de décrire la référence comme allocentrée ou égocentrée, centrée sur l'objet ou sur l'environnement, etc. Concrètement, dans une annotation en XML-TEI ${ }^{25}$, utilisant l'élément $<$ rs $>$ pour le marquage des entités spatiales, nous avions prévu d'employer en ce sens l'attribut @ana, avec les valeurs suivantes : \#RT (relatif à un toponyme), \#RC (relatif à un personnage), \#RA (relatif à un animal), \#ROA (relatif à un objet ou artéfact), \#RSE (relatif à une autre entité spatiale). En outre, le même attribut @ana pouvait recevoir les valeurs \#INC (signale l'inclusion d'une entité de type place dans une autre, comme dans « la cuisine de la maison »), \#PROX et \#DIST (pour les entités décrites comme étant proches ou distantes d'autres : "il passa dans la chambre voisine »), \#ORIG (pour indiquer qu'une entité est le point d'origine d'un mouvement), \#TERM (l'entité constitue le point d'arrivée), \#PASS (l'entité dé- 
signe un lieu de passage : «il entra par la grande porte »), et enfin \#VERT et \#HORZ qui indiquent que l'entité est associée à un mouvement sur la verticale ou l'horizontale (ex. "il regarda en haut»).

Avec ce premier schéma, nous avons tenté une première annotation sur un extrait du roman de Panait Macri, Ioan Tunsu, căpitan de haiduci. Dès le début de l'annotation, nous avons été confrontées au cas particulier des verbes, dont certains jouent un rôle de premier plan dans l'expression de l'espace. Toutefois, donner la consigne d'annoter les entités spatiales avec les verbes dont elles dépendaient posait de nombreux problèmes pratiques, notamment dans le cas des phrases longues, où les ES étaient séparées par plusieurs mots du verbe régisseur. Aussi a-t-il paru plus sage de s'en tenir, dans un premier temps, aux seules expressions nominales, pronominales et adverbiales déjà évoquées.

Par ailleurs, il est rapidement apparu que notre schéma ne permettait pas de rendre compte d'une série d'espaces fréquemment mentionnés par les personnages, mais que l'on pouvait difficilement étiqueter comme " lieu " ou « zone " en raison de leur caractère symbolique et imaginaire. Il s'agit d'expressions comme « l'autre monde », de la désignation des endroits habités en général ( il alla s’installer loin de tout»), de « la tombe " (dans " ce gamin me mettra dans la tombe »), etc. Nous avons donc ajouté une catégorie "topical_space ", susceptible de rendre compte de ces espaces symboliques. En outre, ont donné du fil à retordre certaines expressions désignant des espaces immatériels, le plus souvent liés au corps humain, comme dans "il avait mûri ce projet dans sa tête pendant des mois ${ }^{26}$ ". Après de nombreuses hésitations, et au vu d'autres occurrences problématiques (par exemple certains objets dont le rôle de marqueur spatial n'est pas certain), nous avons créé une catégorie «potential_SE», destinée à accueillir ces différents éléments et permettant de mieux cerner cette dimension métaphorique de l'espace romanesque dont il a été question plus haut.

Le schéma ainsi complété, une seconde tentative d'application a été réalisée, en mobilisant un plus grand nombre de textes et de lecteurs. Outre les deux investigatrices principales du projet $H A I-R O$, quatre autres annotatrices ont été recrutées : une chercheuse de l'université Al. I. Cuza de Iași, qui a d'ailleurs participé à l'élaboration du schéma, et trois étudiantes en lettres et langues, sans connaissances préalables en humanités numériques. Les difficultés qu'elles ont rencontrées lors de l'application du schéma sur d'autres romans ont mené à la décision de l'alléger, en réduisant le nombre d'attributs employés. Seules les valeurs \#RT, \#ROA, \#RC et \#RSE ont été conservées de l'ensemble décrit plus haut - le caractère orientatif ou non orientatif des ES, ainsi que l'embô̂tement des espaces, apparaissant moins immédiatement utiles dans la perspective de l'étude envisagée sur l'espace des haïdouks. À la suite de cet allègement, un guide d'annotation a été élaboré, documentant les types d'entités spatiales retenus ainsi que les valeurs des attributs potentiels.

L'élargissement du groupe d'annotatrices, ainsi que les discussions portées en marge des textes à annoter, ont mis en avant les divergences potentielles dans l'identification et la classification des entités spatiales. Contrairement à ce que suggèrent les études narratologiques, plus intéressées par l'interprétation que par le relevé rigoureux des occurrences, notre expérience nous a amenées à comprendre que ce qui «fait espace » 
diffère considérablement d'un lecteur à un autre. La question est de savoir si, malgré ces divergences, nous pouvons miser sur l'existence d'un socle commun suffisamment large d'entités spatiales que la plupart des lecteurs vont identifier et interpréter de façon consensuelle, ou bien si l'espace, comme les symboles, est un des domaines dans lesquels la subjectivité et la culture encyclopédique du lecteur interviennent le plus lourdement. Dans le premier cas, une annotation " en masse " et une exploitation statistique des résultats sont possibles ; dans le second, c'est plutôt de la confrontation des lectures que peut jaillir l'interprétation. Les outils numériques mobilisés par l'une et par l'autre approche diffèrent, ainsi que la façon de croiser narratologie et numérique.

Quoique éloignée de nos domaines habituels d'intérêt et d'expertise, une mesure de l'accord interannotateurs s'imposait donc, afin de pouvoir prendre les décisions méthodologiques nécessaires pour la suite du projet. On ne visait pas, et on ne s'attendait pas, à atteindre un score élevé d'accord interannotateurs pour cette campagne, qui était à la fois un test de faisabilité et un moment de découverte des outils pour une grande partie de l'équipe. En revanche, cette campagne était susceptible de nous renseigner sur la pertinence de notre schéma pour une approche littéraire, de nous aider à le perfectionner, de mettre en avant des points de vigilance dans la perspective d'une éventuelle automatisation et même de pointer vers des motifs inédits, dont la récurrence n'est pas immédiatement perceptible à une lecture de près.

\section{L'accord interannotateurs et ses enseignements}

Afin de faciliter la tâche des annotatrices et les comparaisons, nous avons décidé de travailler sur des échantillons et d'utiliser un outil facile à prendre en main pour réaliser les annotations.

Dix échantillons de mille mots environ ont été générés, à partir de dix romans d'auteurs différents. L'ensemble des échantillons a été pris en début de roman, une partie a priori riche en indications spatiales, puisque c'est traditionnellement le moment où se met en place la fiction. Chaque échantillon a été proposé à trois annotatrices différentes, en veillant à ce que chaque trinôme comporte au moins une annotatrice plus expérimentée.

Par ailleurs, plutôt que d'annoter en XML-TEI, format que la plupart des annotatrices ne connaissaient pas, nous avons choisi de configurer sur un serveur BRAT qui est un outil dédié à l'annotation, permettant d'importer et d'exporter des formats standards, et favorisant le calcul de l'accord interannotateurs. Un simple soulignement de la chaîne de caractères souhaitée suffit pour annoter, les attributs disponibles et leurs valeurs étant aussitôt proposés sous forme de liste.

Les annotatrices avaient pour consigne de ne pas regarder le travail des autres (les annotations de chacune étant stockées dans des dossiers différents), et de ne pas échanger entre elles sur les difficultés d'annotation rencontrées. Toutefois, il est apparu ultérieurement que les annotatrices de l'équipe roumaine - qui découvraient le logiciel - ont néanmoins échangé sur leurs pratiques d'annotation en raison d'un message d'erreur s'affichant sous BRAT en cas d'entités emboîtées (fi- 
gure 1). Dès lors, l'équipe roumaine a adopté le principe d'une annotation " scindée ». Ainsi, dans l'exemple donné plus bas, "la spatele " (derrière) est étiqueté en tant que "zone », et «bisericii Grecești» (l'église grecque) en tant que toponyme. Selon le guide d'annotation, c'était plutôt le fonctionnement suivant qui était attendu :

FIGURE 1. ANNOTATIONS EMBOÎTÉES SOUS BRAT

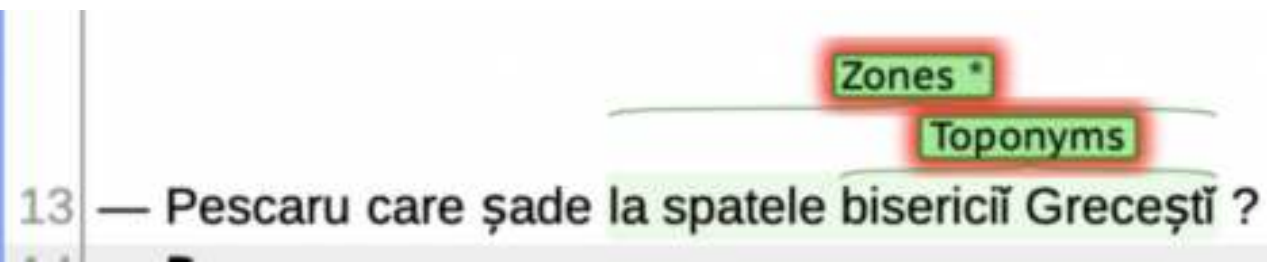

Citation extraite de Ilie Ighel, Moartea banditului Simion Licinsky, roman criminal (București, editura librăriei H. Steinberg, 1891). La phrase à l'écran signifie « Le pêcheur qui habite derrière l'église grecque? ».

Figure produite par les auteurs, licence CC BY

Comme on le verra, cette divergence d'annotation est responsable en bonne partie de la baisse du score d'accord interannotateurs calculé au sujet de nos échantillons.

À l'issue de l'exercice, les annotations ont été comparées à l'aide du module Python Bratutils ${ }^{27}$. Pour chaque trinôme, l'annotatrice la plus expérimentée a été considérée comme produisant l'annotation de référence. Une comparaison des annotations des deux autres annotatrices a été également réalisée, mais un ordre de priorité n'a pas été établi entre les deux. La comparaison a porté sur la délimitation et l'étiquetage des entités spatiales, mais non pas sur l'utilisation des attributs : l'objectif était de constater, en premier lieu, si des lecteurs différents identifient les mêmes suites de mots comme étant porteuses d'information spatiale, et s'ils les catégorisent de la même façon (en tant que toponyme, lieu, zone, etc.).

Les résultats obtenus sont encourageants ; ils suggèrent d'une part, qu'une annotation consensuelle d'un plus vaste corpus est possible, au prix de quelques évolutions du schéma, et d'autre part, que ce dernier est, malgré tout, suffisamment robuste. En effet, malgré des préférences plus marquées pour un tag ou un autre, toutes les annotatrices ont utilisé au moins une fois toutes les étiquettes, ce qui confirme que chacune est utile. Soulignons également qu'aucune annotatrice ne produit des annotations arbitraires (désignées comme "spurious " par le module Bratutils), ce qui suggère qu'elles ne se trouvent pas démunies devant la complexité des expressions spatiales pratiquées dans les romans.

La F-mesure de l'accord interannotateurs ${ }^{28}$ est très variable, avec des scores allant de $10 \%$ à $81 \%$, une moyenne autour de $43 \%$ et la médiane à $44 \%$. Les valeurs les plus élevées sont obtenues quand on compare les membres de l'équipe roumaine entre elles, mais les valeurs au-dessus de $50 \%$ sont plus fréquentes quand on confronte les annotations produites par l'investigatrice principale du côté français avec celles de l'équipe roumaine. Cela est visible dans les deux tableaux suivants (figures 2 et 3 ) : l'un présente les résultats des cinq échantillons annotés exclusivement par l'équipe roumaine, l'autre des échantillons dans lesquels l'annota- 
trice française est intervenue. Comme indiqué plus haut, ce contraste s'explique, au moins dans une certaine mesure, par des différences de pratique sur BRAT au sujet du découpage des entités emboîtées.

Les figures 2 et 3 détaillent les scores utiles au calcul de la F-mesure ou F-score (fsc). Si celle-ci est la moyenne harmonique entre mesures de rappel (rec) et de précision (pre), ces deux mesures sont elles-mêmes le résultat d'opérations utilisant un ensemble d'autres décomptes : " pos " (possible) est le calcul du nombre d'annotations possibles, toutes annotations confondues ; " act " (actual) est le nombre d'annotations de l'annotateur R2 ; "corr» (correct) indique le nombre d'annotations communes aux deux annotateurs, alors que « par " (partially correct) permet de préciser le nombre d'annotations se recouvrant partiellement. En effet, une annotation est d'une part, la sélection d'un ou plusieurs mots du texte et d'autre part, l'attribution d'une étiquette au texte sélectionné. Ainsi " par " comptabilise le nombre d'annotations où seul un des deux critères (les bornes finale et initiale de la sélection ou l'étiquette d'annotation) est strictement identique dans les deux annotations. " inc " (incorrect) et " mis " (missing) listent les annotations incorrectes ou absentes dans l'annotation de l'annotateur $\mathrm{R}^{29}$.

FIGURE 2. RÉSULTATS ÉQUIPE ROUMAINE

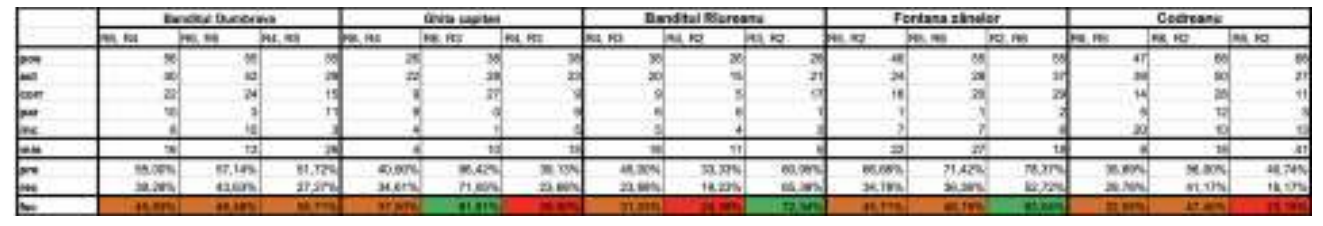

Figure produite par les auteurs, licence CC BY

FIGURE 3. RÉSULTATS ÉQUIPE ROUMAINE VS ANNOTATION FRANÇAISE

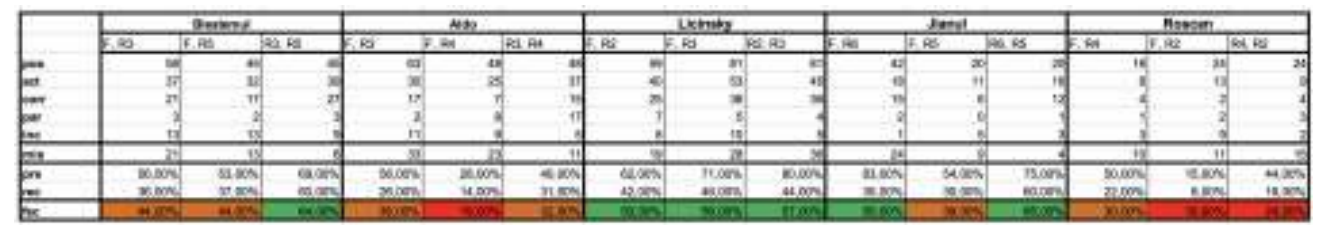

F désigne l'annotatrice française, R2 à R5, les annotatrices roumaines. La différence de pratique de BRAT ayant eu un impact sur les annotations réalisées, comme indiqué plus haut, il nous semble important de souligner ainsi l'origine des différentes annotations.

Figure produite par les auteurs, licence CC BY

Dans une perspective de traitement automatique des langues, ces résultats sont plus faibles que ceux à partir desquels on peut considérer un schéma raisonnablement opératoire pour une campagne d'annotation en masse, et appellent à une analyse des sources les plus communes d'erreur afin de trouver des solutions. Dans la perspective qui est la nôtre, il nous a paru intéressant de mener cette analyse également parce qu'elle livre des enseignements à propos de ces « effets de lecture " de l'espace dont il a été question plus haut.

Bratutils permet des comparaisons de trois types d'annotations (correct/partial/incorrect), mais notre approche permet d'aller au-delà des décomptes, pour revenir aux textes, les mettre en regard et les comparer de visu. Nous sommes reparties des annotations produites par chaque paire d'annotatrices, qui ont été importées et alignées dans une feuille de calcul avec leur numéro respectif, tel qu'assigné par BRAT (T1, 
T2, etc.), le caractère de début et le caractère de fin et, bien entendu, l'étiquette. Trois colonnes ont été ajoutées : dans la première (Difference), nous avons caractérisé les différences constatées, selon un jeu de valeurs qui sera présenté au paragraphe suivant ; dans la seconde (Tag involved), nous avons relevé l'étiquette, ou la paire d'étiquettes concernées ; quant à la troisième, elle sert à indiquer la raison potentielle du désaccord (Reason). Nous donnons plus bas un extrait du fichier de comparaison (figure 4).

À la fois pour la première et pour la troisième des colonnes supplémentaires, nous avons défini un nombre limité de valeurs possibles. On trouvera ainsi dans la colonne "Difference " les valeurs MIS, PRAC et $\mathrm{TAG}^{30}$, pour signaler respectivement les cas où une entité n'est pas du tout signalée par une des annotatrices, les cas où la délimitation des entités n'est pas la même, et enfin les cas où les mêmes jeux d'étiquettes n'ont pas été utilisés. PRAC et TAG peuvent, en outre, se combiner, pour les cas où, à la fois, les limites de l'entité et sa définition seraient divergentes. Ce sont ces cas surtout qui nous intéressent dans une perspective littéraire, dans la mesure où ils donnent à voir des interprétations différentes à partir d'une même base textuelle.

FIGURE 4. COMPARAISON ET CODAGE DES ANNOTATIONS DE ALDO ȘI AMINTA

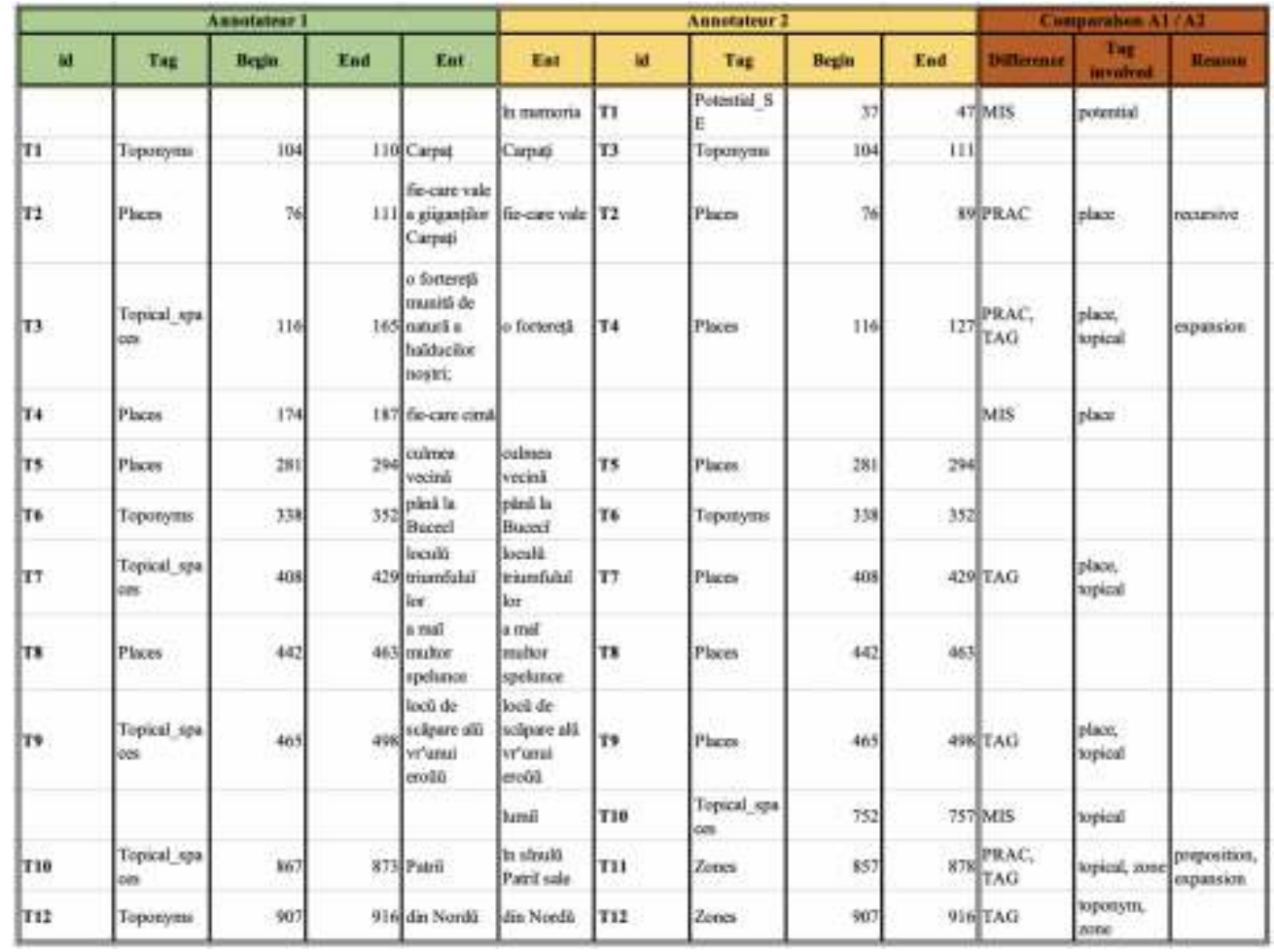

Figure produite par les auteurs, licence CC BY

Dans la colonne "Reason", la liste des valeurs possibles était plus large. On y relevait, en premier lieu, les cas de disparités dus à l'annotation emboîtée $v s$ l'annotation scindée dont il a déjà été question plus haut (code "recursive »). Par ailleurs, après une première observation de la liste nous avons décidé de retenir trois valeurs décrivant les « oublis ", c'est-à-dire les cas où l'entité spatiale n'était pas annotée avec la préposition, le déterminant ou l'expansion qui l'accompagnent dans le texte (codes "preposition", «determiner " et "expansion»). Trois autres valeurs signalaient si l'entité spatiale posant problème à l'une ou l'autre des annotatrices était en rapport avec le corps humain («body»), en lien avec la 
position d'un personnage ( character») ou en lien avec un objet ou composant d'objet («object»); ces trois cas avaient, en effet, suscité pas mal de discussions lors de l'élaboration du schéma d'annotation, et avaient mené à la création du code "Potential-SE ". Un code supplémentaire a également été proposé dans un premier temps, "archaism ", pour signaler les oublis ou les erreurs d'étiquetage liés à l'emploi de mots inexistants dans le roumain moderne ("olac", voiture de poste ou service de messagerie ; "tactul vistieriei ", l'hôtel de la trésorerie), mais sa contribution s'est avérée plus que modeste, même si les romans composant notre corpus sont écrits dans une langue parfois assez archaïsante. À retenir également que les colonnes "Difference " et "Tag involved sont censées être systématiquement remplies en cas de divergence d'annotation, alors que la colonne "Reason" peut rester vide : comme indiqué plus haut, les deux premières colonnes ont été conçues dans la perspective d'une amélioration du schéma pour une annotation "en masse ", tandis que le but de la troisième est plutôt de fournir des pistes de compréhension du travail du texte, tel qu'il est fait lors de lecture par chaque réceptrice, sans avoir la prétention de tout expliquer.

Compte tenu du caractère non trivial du typage des différences d'annotation, et du temps que l'opération a demandé, elle n'a concerné que cinq échantillons parmi les dix, à savoir ceux dans lesquels l'annotatrice française était intervenue. Les résultats obtenus confirment qu'une annotation globale des entités spatiales romanesques est envisageable, quitte à retravailler certains aspects du schéma proposé plus haut. Certes, le taux d'accord observé ainsi «tombe " à seulement 27,62 \% ${ }^{31}$, mais on peut considérer qu'une meilleure explication de l'exercice permettrait d'éliminer, ou de réduire fortement, les 18,47 \% de disparités en termes de pratique, ce qui rapprocherait fortement l'accord interannotateurs d'un seuil d'acceptabilité que l'on pourrait fixer à $50 \%$. En effet, plus de la moitié des cas de disparités dans le découpage sont, en effet, liés à la notation emboîtée ou scindée pratiquée par les différents membres de l'équipe. La cause la plus fréquente est ensuite l'oubli des prépositions ( $23 \%$ des cases PRAC), puis des expansions $(18,92 \%)$ et enfin des déterminants (3,38\%). Un guide d'annotation plus riche, fournissant de nombreux exemples d'empans de texte à annoter, devrait permettre de mieux sensibiliser les annotateurs à ces aspects. Nous prévoyons, par ailleurs, de fournir à tous un petit texte d'entraînement, accompagné des solutions attendues, ce qui devrait également améliorer la précision du découpage.

Il faut aussi noter que les divergences les plus fréquentes $(32,78 \% \mathrm{du}$ total des annotations, ou $45 \%$ du total des désaccords) proviennent des entités manquantes, c'est-à-dire annotées seulement par l'une ou par l'autre des annotatrices d'un même texte ; les réelles divergences d'étiquetage ne concernent, elles, qu'un peu plus de $20 \%$ du total des annotations, pourcentage obtenu en additionnant les cases marquées "TAG » et «PRAC, TAG ». Corrélé au nombre d'annotations entièrement correspondantes, cela signifie que, lorsque les annotatrices identifient toutes les deux une entité spatiale, elles s'accordent spontanément quant à sa nature dans $69,25 \%$ des cas, et sont plus rarement en désaccord quant à l'étiquette à lui attribuer. 
Quant aux annotations manquantes, il est instructif d'analyser la contribution respective de chaque type d'étiquette à ce sous-ensemble, telle que résumée dans le tableau 1.

TABLEAU 1. ÉTIQUETTES DES ENTITÉS MANQUANTES

\begin{tabular}{|l|l|l|l|l|}
\hline $\begin{array}{l}\text { Effectif } \\
\text { total }\end{array}$ & Type & $\begin{array}{l}\text { Nombre d'entités } \\
\text { manquantes }\end{array}$ & $\begin{array}{l}\text { Pourcentage d'entités } \\
\text { manquantes }\end{array}$ & $\begin{array}{l}\text { Pourcentage } \\
\text { d'effectif }\end{array}$ \\
\hline 10 & Address & 1 & $0,51 \%$ & $10 \%$ \\
\hline 35 & Path & 6 & $3,05 \%$ & $17,14 \%$ \\
\hline 384 & Place & 62 & $31,47 \%$ & $16,15 \%$ \\
\hline 70 & $\begin{array}{l}\text { Poten- } \\
\text { tial }\end{array}$ & 45 & $22,84 \%$ & $64,29 \%$ \\
\hline 124 & Topical & 45 & $22,84 \%$ & $36,29 \%$ \\
\hline 88 & $\begin{array}{l}\text { Topo- } \\
\text { nym }\end{array}$ & 3 & $1,52 \%$ & $3,41 \%$ \\
\hline 22 & Vehicle & 8 & $4,06 \%$ & $36,36 \%$ \\
\hline 232 & Zone & 27 & $13,71 \%$ & $11,64 \%$ \\
\hline
\end{tabular}

Il en résulte que la plus grande partie des annotations manquantes concerne des entités spatiales potentielles, ou " topiques ", dont l'identification est, par nature, plus délicate. Certes, les «places » non identifiées par l'une ou l'autre des annotatrices forment un ensemble numériquement significatif, voire saillant, mais cela doit être pondéré par l'observation que cette catégorie est la plus employée dans l'ensemble de nos échantillons. Sans prendre en compte les cas où ces "places " n'en sont peut-être pas (comme les "rames des fenêtres » [chenare] identifiées par une annotatrice, ou le "cartable " [ghiozdan], qui passe de main en main dans la nouvelle Iancu Jianul, avant de servir une fois d'oreiller de fortune), observons que seulement 16,15\% des "places " (sur l'ensemble des possibilités) ne sont pas vues par une ou l'autre des annotatrices, alors que la proportion monte à $64 \%$ dans le cas des entités potentielles.

Des observations concordantes peuvent être faites au sujet des désaccords d'étiquetage. On trouvera, dans le tableau 2, toutes les paires d'étiquettes concernées, certaines de façon très anecdotique. 


\begin{tabular}{|l|l|l|}
\hline Combinaisons & Nombre & Pourcentage de TAG et PRAC, TAG \\
\hline Address, zone & 5 & $4,07 \%$ \\
\hline Path, place & 4 & $3,25 \%$ \\
\hline Path, topical & 6 & $4,88 \%$ \\
\hline Path, zone & 1 & $0,81 \%$ \\
\hline Place, potential & 1 & $0,81 \%$ \\
\hline Place, topical & 38 & $30,89 \%$ \\
\hline Place, toponym & 4 & $3,25 \%$ \\
\hline Place, zone & 34 & $27,64 \%$ \\
\hline Potential, topical & 7 & $5,69 \%$ \\
\hline Potential, zone & 2 & $1,63 \%$ \\
\hline Topical, zone & 15 & $12,20 \%$ \\
\hline Toponym, zone & 4 & $3,25 \%$ \\
\hline Vehicle, zone & 2 & $1,63 \%$ \\
\hline
\end{tabular}

De nouveau, on remarque la contribution importante de l'étiquette "topical_space " à l'ensemble des divergences ; toutes combinaisons confondues ("path, topical ", " place, topical ", etc.), on arrive à plus de $51 \%$ des cas. Curieusement, quand les entités spatiales sont utilisées dans des figures de style, elles semblent ne plus être perçues comme telles : c'est le cas dans des comparaisons de toute sorte («Unŭ mititelu palatŭ, ce avea forma degenerată a unu ̌ castelŭ din evulŭ medianŭ " : " un petit palais, qui avait la forme dégénérée d'un château du Moyen Âge "), des métaphores ( fiecare vale a giganților Carpați era ofortereță munită de natură a haŭducilor noștri »: " chaque vallée des gigantesques Carpates était une forteresse donnée par la nature à nos haïdouks "), des hyperboles ("se asvîrleaŭ titanice pînă la nuorĭ " : " se jetaient jusqu'aux nuages $\left.{ }^{32} »\right)$, etc. Cela peut être lié aux conditions mêmes de l'exercice, qui sursollicite la conscience référentielle des lectrices et les amène, dès lors, à ne pas considérer comme porteuses d'une information spatiale les expressions ne renvoyant pas immédiatement à un objet localisable dans le monde réel. Quoi qu'il en soit, cela nous invite à repenser la façon dont nous introduisons ces « espaces topiques » dans le guide d'annotation, afin de stimuler la perception de l'espace conceptuel, autant que de l'espace factuel (Westphal 2007).

En observant les paires d'étiquettes en désaccord, les étiquettes entre lesquelles il y a le plus de flottement sont " place » et «zone », d'une part, et entre "place " et "topical ", d'autre part. Dans les deux cas, on peut parler d'un biais en faveur de "place " de la part des annotatrices roumaines, par comparaison avec l'annotatrice française ; "place " semble ainsi remplir la fonction d'un tag "par défaut ", ce qui empêche, dans certains cas, une analyse plus fine de l'entité. Cette confusion est gênante dans la perspective de la question initiale de recherche, qui nous avait amenées à nous demander si on peut identifier dans les romans une tendance à l'utilisation de "places » en lien avec les personnages des paysans, et de «zones " en lien avec les haïdouks, ce qui tendrait à confirmer l'hypothèse que le personnage est un vecteur de liberté. Aussi nous 
semble-t-il important d'affermir la distinction entre "place " et "zone ", en recommandant que toutes les entités au pluriel (ou presque) soient considérées comme des zones, et non pas des lieux. Le traitement d'entités comme « les sommets alentour » ou «dans plusieurs régions du Soleil Levant " devrait ainsi être facilité. Cela n'empêchera pas, en revanche, l'hésitation à propos de certaines entités comme "chez moi ", "la forêt " dans " en passant par la forêt ", " la cour ", " le jardin public ", à propos desquelles il n'est pas toujours facile de décider si elles sont évoquées comme des points de repère sur une carte imaginaire (ce qui inviterait à les étiqueter plutôt en tant que "place»), ou bien comme des contenants de plusieurs points " géolocalisables " (donc «zone »). L'évaluation du caractère ouvert ou limité de l'horizon des personnages n'a pas vocation à être rigoureusement exacte, et il faudra rester conscient, lors de l'analyse, qu'en ce qui concerne les entités spatiales romanesques un désaccord d'au moins $30 \%$ continuera à subsister entre les différents récepteurs, toutes catégories d'étiquettes confondues.

\section{En guise de conclusion : quelques observations sur l'espace des haïdouks}

Au terme de ce parcours, il est sans doute trop tôt pour tenter de répondre à la question de recherche initiale. Les échantillons permettent toutefois de formuler quelques observations et de suggérer plusieurs pistes.

Les 1420 annotations produites, toutes annotatrices confondues, connaissent une répartition très contrastée entre les dix romans (figure 5), traduisant ainsi d'importantes différences stylistiques dans la mise en place du cadre. Chaque échantillon ayant été annoté trois fois, cela donne une moyenne de 47 annotations par texte, mais le nombre le plus bas d'annotations pour un échantillon descend à 18 , tandis que le nombre le plus élevé atteint 90. Visiblement, certains auteurs " saturent » l'incipit de références spatiales, tandis que d'autres s'en tiennent à une sorte d'essentiel. Il sera intéressant d'observer, par la suite, si ce contraste se maintient à l'échelle des romans, ou bien si le retard quantitatif de certains est " rattrapé " dans les chapitres ultérieurs, et de quelle façon. De même, au fur et à mesure de l'annotation d'autres romans des mêmes auteurs, on pourra évaluer si la "pauvreté " initiale de l'espace est idiosyncrasique, ou bien un choix narratologique lié à un certain type d'incipit. 


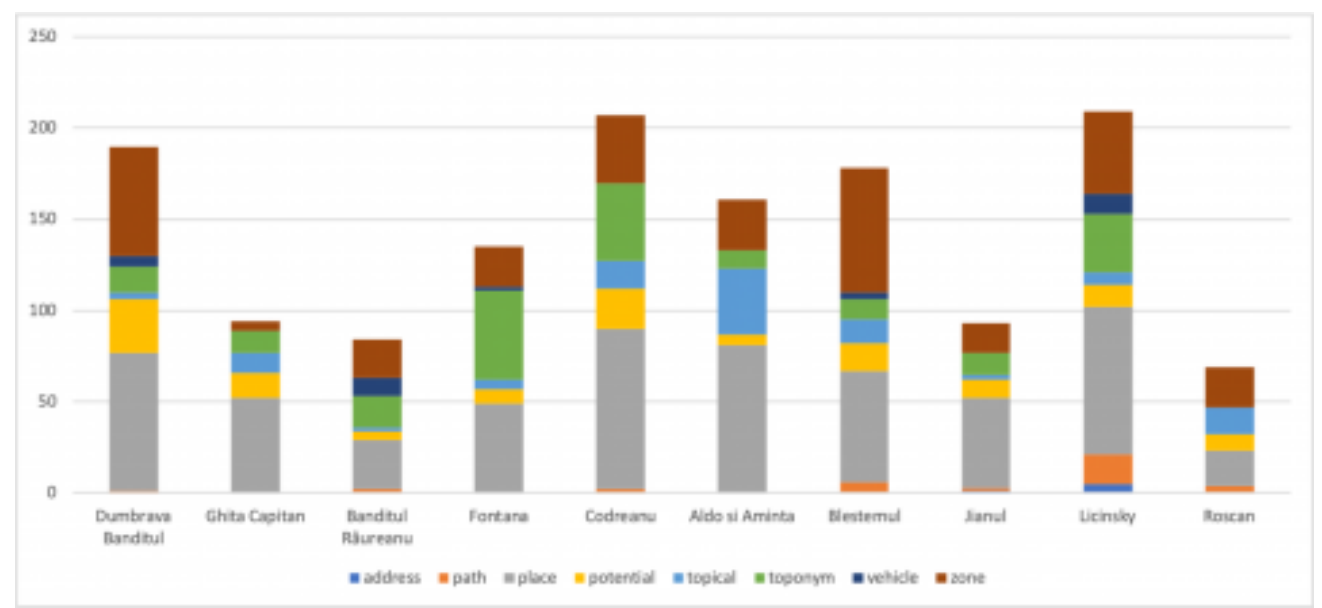

Figure produite par les auteurs, licence CC BY

Même s'il faut les prendre avec précaution, compte tenu des fragilités décrites plus haut, les tendances d'utilisation des entités spatiales, telles que suggérées par cette figure 5, s'avèrent assez éclairantes. Remarquons d'abord que les entités de type «topical » et «potential» ont la même fréquence d'utilisation que les toponymes, ce qui met plus clairement en perspective les limites de la contribution de ces derniers à la mise en place du cadre narratif. Si certains romans, comme Codreanu ou Fontana zânelor, bénéficient d'un ancrage géographique plus ferme, d'autres se contentent de quelques points de repère ou s'en passent même entièrement - comme dans le cas de Roșcan. S'il n'est pas question de minimiser l'intérêt des approches actuelles de la géographie des œuvres littéraires, fondées en grande partie sur des outils de reconnaissance et résolution d'entités nommées, ces chiffres et ces observations suggèrent les dimensions du continent que de tels outils laissent dans l'ombre.

Allant un peu à l'encontre de nos attentes, les zones ne sont pas les plus nombreuses dans les romans à haïdouks. Toutefois, comme on l'a vu plus haut, cela peut être lié à un biais en faveur des "places ", plus faciles à conceptualiser. Malgré tout, les «zones " viennent en deuxième position en termes de fréquence, et elles forment des groupes particulièrement significatifs dans Blestemul, Dumbravă Banditul, Licinsky ou Codreanu. Au moins dans ces œuvres-ci, il sera intéressant d'observer les associations qui se créent entre zones, places et types de personnages, dans la perspective de notre postulat selon lequel une tension existe, dans nos romans, entre l'espace des haïdouks, caractérisé par l'ouverture et la mobilité, et celui des paysans, prédominant dans la Roumanie rurale $d u \mathrm{XIX}^{\mathrm{e}}$ siècle et du début $\mathrm{du} \mathrm{Xx}^{\mathrm{e}}$ siècle, et définissable en tant que limité et structuré par des relations claires.

Même si elles donnent lieu à un nombre plutôt réduit d'annotations, et qu'elles manquent entièrement dans la moitié des cas (Ghiță Căpitan, Codreanu, Aldo și Aminta, Jianul, Roșcan) les catégories " path " et «vehicle» sont particulièrement intéressantes, car dotées d'un véritable pouvoir discriminant entre les romans. Les échantillons Licinsky et Banditul Râureanu s'avèrent ainsi très différents des autres de ce point de vue, avec une préférence marquée pour les déplacements. Peut-être ce tropisme - qui reste à confirmer sur un plus large corpus - est-il lié à la différence éthique entre les héros de ces romans, tous les deux qualifiés de "bandits " (brigands) et ne semblant pas partager la même révolte 
contre des inégalités sociales que des personnages éponymes comme Ghiță Căpitan ou Codreanu - dans les échantillons desquels on ne trouve aucune mention de chemin ou de véhicule. La manipulation de l'espace, et probablement les valeurs qui lui sont associées, seraient ainsi différentes selon que le personnage principal est un authentique haïdouk, ou un hors-la-loi plus proche du régime du droit commun, si on peut dire. À plus grande échelle, de telles observations promettent d'intéressants regroupements entre des romans plutôt "statiques ", qui enchaînent les scènes et les résumés, et des romans "dynamiques ", où les déplacements prennent de l'importance. À noter également que l'une comme l'autre étiquette sont très peu impliquées dans les trois types de divergences constatées (entités manquantes, désaccords d'étiquette, découpages différents), restant en dessous de $5 \%$ de contribution par rapport à chacune de ces populations.

L'expérience d'annotation des entités spatiales présentée ici est encore en cours de déploiement. La mesure de l'accord interannotateurs s'est avérée suffisamment satisfaisante pour continuer l'exercice, mais elle a aussi attiré notre attention sur la nécessité de revoir nos catégories et nos consignes. Elle nous a surtout permis de mieux prendre la mesure de la complexité de l'inscription de l'espace dans les œuvres littéraires et de la visibilité très contrastée des entités spatiales pour les lecteurs. La rencontre entre une intuition critique et la rigueur du numérique s'est ainsi avérée féconde, nous amenant à la fois à faire évoluer nos perspectives conceptuelles et interprétatives, et à prendre clairement conscience de ce que l'ordinateur peut et ne peut pas lorsqu'il est question d'explorer des œuvres littéraires.

\section{Bibliographie}

Aurnague, Michel. 2012. "De l'espace à l'aspect : les bases ontologiques des procès de déplacement ». Corela HS-12 (décembre). https://doi.org/10.4000/corela.2846.

Bachelard, Gaston. 2020 [1957]. La Poétique de l'espace. Paris : PUF.

Blanchot, Maurice. 1988 [1955]. L’Espace littéraire. Paris : Gallimard.

Bodenhamer, David J., John Corrigan et Trevor M. Harris. 2010. The Spatial Humanities : GIS and the Future of Humanities Scholarship. Bloomington : Indiana University Press.

Bodenhamer, David J., John Corrigan et Trevor M. Harris. 2015. Deep Maps and Spatial Narratives. Bloomington et Indianapolis : Indiana University Press.

Camus, Audrey. 2011. "Chapitre II. Espèces d'espaces : vers une typologie des espaces fictionnels ». Dans Topographies romanesques, édité par Audrey Camus et Rachel Bouvet, 3344. Rennes : Presses universitaires de Rennes. https://doi.org/10.4000/books.pur.38348.

Dicționarul cronologic al romanului românesc. 2003. București : Editura Academiei Române.

Kolarz, Walter. 2003. Mituri și realități în Europa de Est. Iași : Polirom.

Jackendoff, Ray. 1983. Semantics and Cognition. Cambridge : MIT Press.

Leersen, Joep. 2010. "The Rural Outlaws in East-Central Europe ». Dans History of the Literary Cultures of East-Central Europe, vol. 4. Types and Stereotypes, édité par Marcel CornisPope et John Neubauer, 407-440. Amsterdam, Philadelphia : John Benjamins.

Levinson, Stephen C. 2014. Space in Language and Cognition. Explorations in Cognitive Diversity. Cambridge : Cambridge University Press.

Mazilu, Dan-Horia. 2016. Lege și fărădelege în lumea românească veche. Iași : Polirom.

Nedelcu, Veronica. 2013. Adverbul ca modalitate de exprimare a locului și timpului în limba română. Bucureşti : Editura Universitară. 
Patraș, Roxana. 2019. "Hayduk Novels in the Nineteenth-Century Romanian Fiction : Notes on a Sub-Genre ». Swedish Journal of Romanian Studies 2 (1) : 24-33. https://journals.lub.lu.se/sjrs/article/view/18769.

Patraș, Roxana, Lou Burnard et Carolin Odebrecht. 2019. « COST-ELTeC/ELTeC-rom : Release with 24 Novels at Level 1. » Zenodo. https://doi.org/10.5281/ZENODO.3543112.

Pavel, Thomas. 1988. L'Univers de la fiction. Paris : Seuil.

Perec, Georges. 1974. Espèces d'espaces. Paris : Galilée.

Pustejovsky, James et Kiyong Lee. 2017. " Enriching the Notion of Path in ISO-Space ". Dans Proceedings of the Thirteenth Joint ACL-ISO Workshop on Interoperable Semantic Annotation (ISA-13). Montpellier, September 19, édité par Harry Bunt, 134-139. Tilburg Center for Cognition and Communication, Tilburg University.

Pustejovsky, James, Kiyong Lee et Harry Bunt. 2019. « The Semantics of ISO-Space ». Dans Proceedings of the 15th Joint ACL-ISO Workshop on Interoperable Semantic Annotation (ISA15). Gothenburg, May 23, édité par Harry Bunt, 46-53. Tilburg Center for Cognition and Communication, Tilburg University.

Raveca-Buleu, Constantina. 2011. Paradigma puterii în secolul al XIX-lea. Bucureşti : Ideea Europeană.

Richardson, Andrew. 2019. «Creative Experiments into the Spatial Visualisation of Literary Texts ». Communication présentée au ADHO Geohumanities SIG Conversations Workshop, Uthrecht, 9 juillet. https://geohumanities.org/?p=115.

Stadler, Jane, Peta Mitchell et Stephen Carleton. 2016. Imagined Landscapes : Geovisualizing Australian Spatial Narratives. Bloomington : Indiana University Press.

Svorou, Soteria. 1994. The Grammar of space. Amsterdam, Philadelphia : John Benjamins.

Taylor, Joanna et Christopher Donaldson. 2019. « Footprints in Spatial Narratives : Reading the Limits of Digital Literary Mapping ». Communication présentée au ADHO Geohumanities SIG Conversations Workshop, Uthrecht, 9 juillet. https://geohumanities.org/? $\mathrm{p}=115$.

Thiesse, Anne-Marie. 2019. La Fabrique de l'écrivain national : entre littérature et politique. Paris : Gallimard.

Vandeloise, Claude. 1986. L'Espace en français. Sémantique des prépositions spatiales. Paris : Seuil.

Vătavu, Bogdan-Vlad. 2014. « Boierii şi haiducii : De la duşmănie la cârdăşie - The Boyars and the Haiduks : From Enmity to Collaboration ». Dans Seminatores in artium liberalium. Agro studia in honorem et memoriam Barbu Ștefănescu, 81-91. Bucureşti : Editura Academiei Române - Editura Muzeului Țării Crişurilor.

Westphal, Bertrand. 2007. La Géocritique. Réel, fiction, espace. Paris : Éditions de Minuit.

\section{Annexe}

LISTE DES ROMANS INCLUS DANS LE CORPUS HAI-RO

Anonim (MCP), s.l.n.d. Roșcan Haiducul.

Anonim, s.l.n.d. Banditul Grozea cu cei 12 bandiți care a omorît 20 de femei.

Anonim, s.l.n.d. Gruia şi Mereanu. Cei mai vestiți căpitani de haiduci.

Anonim, s.l.n.d. Haiducul Jianu-Lupul şi Isprăvile lui Cerceluş.

Anonim, s.l.n.d. Groasnicii bandiții Lache şi Bâbeanu.

Anonim, s.d. Ruxanda haiduciasa. București : Concurența.

Anonim (D.D.P), 1882. Codreanu, mare haiduc național. București : Editura Typo litografia Dor. P. Cucu.

Bălănescu, Simeon, 1894. Blestemul. Poveste născocită. București : Stabilimentul Grafic I. V. Socec. 
Bălănescu, Simeon, 1895. Sfârşitul blestemului. București : Tip. Gutenberg Joseph Göbl.

Baronzi, George, 1894. Mina haiduceasa, fata codrilor. Nuvelă originală. București : Editura şi Tipo litografia P. Cucu.

Baronzi, George, 1896. Fontana zânelor. București : Editura şi Tipo Litografia Dor. P. Cucu.

Boerescu, Costache, 1855. Aldo şi Aminta sau Bandiții. București : Tip. Bisericească din Sf. Mitropolie.

Dragoslav, Ion, s.l.n.d. Codreanu Haiducu.

Dumbravă, Bucura, 1908. Haiducul. București : Editura Librăriei Şcoalelor C. Şteflea.

Dumbravă, Bucura, 1912. Pandurul. București : Editura Librăriei Şcoalelor C. Şteflea.

Ighel, Ilie, 1891. Moartea banditului Simion Licinsky. Roman criminal. București : Editura Librăriei H. Steinberg.

Ighel, Ilie, 1892. Dragoş, hoțul Tecucilor. București : H. Steinberg.

Ighel, Ilie, 1892. Tâlharul Fulger. Roman criminal întâmplat în timpul revoluției de la 1848. București : Editura Librăriei H. Steinberg.

Igiroşanu, Horia, s.l.n.d. Ciocoii. Roman după filmul românesc relizat şi conceput de Horia Igiroşanu.

Istrati, Panait, 1930. Haiducii. București : Cugetarea.

Istrati, Panait, 1937. Domnița din Snagov. București : Cartea Românească.

Lazăr, s.l.n.d. Haiducul Graur sau Mihnea Banul Olteniei.

Macri, Panait, 1883. Ghiță Cătănuță, căpitan de haiduci. Nuvelă originală. București : Editura Tipo Litografiei Dor. P. Cucu.

Macri, Panait, 1887. Ioan Tunsu, căpitan de haiduci. București : Cultura Românească.

Macri, Panait, 1894. Haiducul Țandură, Scriere originală cu adaose şi modificări. București : Editura Librăriei H. Steinberg.

Macri, Panait, 1899. Bostan, haiduc de peste Milcov. București : Tip. Providența.

Marcu, Anton, s.l.n.d. Codreanu Haiducul. Nuvelă originală.

Marcu, Anton, s.l.n.d. Ghiță Cătănuță, căpitan de haiduci.

Mătasă, C., 1939. Movila haiducului. București ; Cartea Românească.

Munte Stânceanu, Alexandru, s.l.n.d. Graur Vulpoiu, Grozavul Căpitan de haiduci.

Pelimon, Alexandru, 1855. Hoții şi hangiul. Bucureşti : Tip. Bisericească din Sf. Mitropolie.

Popescu, N.D., 1873. Iancu Jianul, căpitanul de haiduci. București : Editura Librăriei H. Steinberg.

Popescu, N.D., 1881. Miul haiducul. O pagină din epoca fanarioților. București ; Editura Librăriei H. Steinberg.

Popescu, N.D., 1881. Tunsul haiducul. Nuvelă originală. București : Editura Librăriei H. Steinberg. 
Popescu, N.D., 1882. Codreanu haiducu. Nuvelă originală. București : Editura Librăriei H. Steinberg.

Popescu, N.D., 1887. Iancu Jianu, zapciu de plasă. Nuvelă originală. București : Editura Librăriei H. Steinberg.

Popescu, N.D., 1892. Bujor, căpitan de haiduci. București : Editura Librăriei H. Steinberg.

Popescu, N.D., 1892. Boierii haiduci. Nuvelă originală. București : Editura Librăriei H. Steinberg.

Popescu, N.D., 1893. Radu Anghel. Nuvelă originală. București : Editura Librăriei H. Steinberg.

Popescu, N.D., 1894. Moartea lui Iancu Jianu căpitanul de haiduci. București : Editura Librăriei H. Steinberg.

Popescu, N.D., s.l.n.d. Aga Gruia Grozovean.

Popescu, N.D., s.l.n.d. Corbea Haiducul în închisoare.

Popescu, N.D., s.l.n.d. Iancu Jianu haiducul.

Popescu, N.D., s.l.n.d. Tunsu căpitan de haiduci.

Popescu Panait, s.l.n.d. Vestitul Bandit Dragoș.

Popescu Panait, s.l.n.d. Groza Cațaonilor, Grozavul căpitan de haiduci (Fulger).

Rădulescu G., 1891. Banditul Rîureanu sau Un înger în infern, s. 1. Editura Librăriei române Filip Lazăr.

Rădulescu-Niger, N., 1893. Căpitanul Ropotă. București : Editura Librăriei Socec \& Comp.

Rosetti, Radu, 1905. Cu paloșul, s. 1.

Stoenescu, Th. M., 1892. Drăguşin haiducul. Istorie adevărată. Braşov : Editura Librăriei Ciurcu.

Stoenescu, Ştefan, 1892. Corbea. Istoria originală. Braşov : Editura Librăriei Ciurcu.

\section{Notes}

1 Il est important de rappeler qu'aux XVIII ${ }^{\mathrm{e}}-\mathrm{XIX}^{\mathrm{e}}$ siècles l'Empire ottoman tient sous sa domination une bonne partie de l'Europe du Sud-Est, les pouvoirs régaliens étant exercés dans ces pays, soit directement par des hauts fonctionnaires turcs, soit par l'intermédiaire de représentants issus de minorités nationales et nommés par la Haute Porte. Dans les deux principautés roumaines concernées par ce phénomène (la Transylvanie se trouvant sous domination austro-hongroise), ces représentants sont des Grecs du quartier Phanar à Constantinople - ce qui explique la fréquence de l'apparition, dans les romans à haïdouks, de Grecoteï et de Phanariotes avec lesquels les haïdouks auront maille à partir.

2 Pendant la période communiste, il est même présenté comme le représentant du prolétariat luttant contre les classes dominantes. Pour une présentation plus détaillée de ces différents aspects, voir Thiesse (2019), Vătavu (2014), Mazilu (2016), Raveca-Buleu (2011), Patraș (2019).

3 Il participe ainsi à la construction du mythe national de la résistance roumaine aux vicissitudes de l'histoire (Kolarz 2003).

4 Défini comme ensemble de places et d'objets cooccurrents qui constituent le cadre d'une ou plusieurs actions, pendant une période de temps donnée. 
5 Notons cependant qu'aucun des systèmes de NER («named entities recognition ») existants ne fonctionne de façon optimale pour le roumain, langue généralement peu outillée dans une perspective de traitement automatique des langues.

6 Voir en ce sens la communication d'Andrew Richardson (2019) ou celle de Joanna Taylor et Christopher Donaldson (2019).

7 Citons le projet, déjà plus ancien, de cartographie de la République des Lettres du xviII ${ }^{\mathrm{e}}$ siècle (http://republicofletters.stanford.edu), l'Atlas littéraire de l'Europe (http://www.literaturatlas.eu/en/index.html) ou bien les travaux de Stadler, Mitchell et Carleton (2016) sur la représentation de l'espace australien dans la narration et sa visualisation grâce au géoréférencement, ou encore les réflexions sur l'exploitation des marqueurs géospatiaux et les métaphores pour la conversion du texte non structuré en données SIG structurées de Bodenhamer, Corrigan et Harris 2010).

8 Qu'elle soit effectuée dans le cadre d'une édition XML-TEI des textes ou à l'aide d'outils d'annotation en ligne, comme BRAT, dont il sera de nouveau question dans ce qui suit.

9 Comme NooJ (http://explorationdecorpus.corpusecrits.huma-num.fr/nooj/) ou Unitex ( https://unitexgramlab.org/fr/). Les prépositions locatives étant plutôt bien spécialisées en roumain, malgré certaines superpositions avec des adverbes locatifs (Nedelcu 2013), et le vocabulaire de l'espace étant relativement spécialisé, il est tout à fait envisageable de recourir à de tels outils. Toutefois, l'un et l'autre systèmes ont été peu appliqués au roumain, et leur utilisation demanderait un étalonnage préalable, dont la durée prévisible excède largement celle du projet dans lequel cet article est ancré.

10 De type entraînement supervisé à partir d'un corpus gold ou système BERT (Bidirectional Encoder Representations from Transformers).

11 Nous mettons ce terme entre guillemets car il est évident qu'une annotation manuelle, même démultipliée grâce à du crowdsourcing, ne produira pas autant d'observables qu'un automate.

12 Létablissement d'une telle carte est un des objectifs du projet HAI-RO (voir plus bas pour une description du projet). Sa réalisation dépend de l'achèvement en 2021 de la numérisation de la collection.

13 En anglais, «inter-annotators agreement », en abrégé IAA.

14 https://www.distant-reading.net.

15 Pour une description du projet, voir https://proiectulbrancusihairo.wordpress.com. Le projet réunit des membres du laboratoire Lattice (UMR 8094), qui sont désignés dans ce texte comme "l'équipe française », et des membres de l'université Al. I. Cuza de Iasi, désignés comme «l'équipe roumaine ».

16 Il s'agit de la collection roumaine de l'European Literary Text Collection (ELTeC) en cours de construction au sein de l'action COST Distant Reading. Pour connaître les principes de constitution et l'état actuel d'achèvement de la collection roumaine, voir : https:// distantreading.github.io/ELTeC/rom/index.html?fbclid=IwAR0ZlaQJPGI4dpg7VQ5GliAo4nxdgwLrIKNzAfO543DFSEf1PwdVh5xa8/.

17 Toponymes, comme "Bucarest », « Tecuci », etc. ; noms communs de lieux (deal [colline] ; câmpie [plaine] ; rîu [rivière], etc.) ; noms communs devenus noms propres ou parties de noms propres (prund, sable mêlé de gravier se trouvant au fond des rivières, et étant devenu le nom de plusieurs villages en Roumanie ; obor, cour pour les animaux, ayant donné le nom à un quartier de Bucarest, etc.).

18 Notamment des prépositions locatives, en roumain (la [à], spre [vers]), le suffixe -ești, signalant une entité nommée comme étant probablement un nom de lieu (Belcești, Marașești), etc.

19 Voir aussi Pustejovsky, Lee et Bunt (2019).

20 Les auteurs se réfèrent ici, probablement, aux spécialistes des sciences du texte qui adoptent une interprétation subjective et perceptive, postkantienne, de l'espace et du temps ; une telle approche est à l'œuvre, par exemple, dans Blanchot (1988), Bachelard (2020), Perec (1974), Pavel (1988).

21 Pour une perspective complémentaire sur la « résistance » de l'espace de fiction au rattachement à la géographie réelle, et une tentative subséquente d'établissement d'une typologie, voir Camus (2011).

22 Les référents spatiaux romanesques (et plus largement culturels) apparaissent ainsi " stratifiés ", ce qui invite à l'adoption d'une démarche de "deep mapping ", en combinant de la fouille de données géolocalisées et les informations issues de sources humanistes. 
23 Les références relatives donnent, dans un second temps, lieu à une subdivision : référence absolue (« il habite Paris »), une référence linguistique explicitée de façon anaphorique ou cataphorique ( il habite au Sud de Paris»), et une référence pragmatique, liée au contexte de communication (" il habite ici»).

24 Nos définitions s'inspirent des travaux linguistiques sur le fonctionnement des prépositions, à la fois de celles qui distinguent entre lieu et région (Jackendoff 1983), et de celles qui s'écartent de cette bipartition pour la description des procès de déplacement (Vandeloise 1986). On reconnaîtra ainsi dans notre définition du lieu des échos clairs de Aurnague (2012) (" un lieu est une entité fixe/stable (+fix) dans un cadre de référence, à laquelle est associée une portion d'espace immatériel ", p. 2), mais on mesurera également la distance que nous prenons avec cette source dans l'établissement de ce qu'est une « zone ", où l'on couvre plusieurs des phénomènes mentionnés plus haut (référence linguistique et pragmatique par différents moyens).

25 Pour mémoire, la collection $H A I-R O$ est conçue dans ce format, qui présente l'avantage d'assurer l'expressivité à long terme de l'annotation pratiquée, et son utilisabilité dans le cadre d'autres projets. Toutefois, pour des raisons pragmatiques exposées dans ce qui suit, la suite de l'annotation a été menée dans un autre format.

26 Comme remarqué par un des relecteurs de cet article, que nous remercions, la même idée peut être rendue par "il y avait pensé pendant des mois ". Il est clair que l'utilisation d'une entité spatiale n'est pas imposée par la langue, mais relève d'un choix. Cela plaide dans le sens du relevé de telles occurrences.

27 Module développé par Aleksandar Savkov et disponible à cette adresse : https://github.com/savkov/bratutils/.

28 La F-mesure, ou F-score (fsc), est la moyenne harmonique des mesures de rappel (rec) et de précision (pre) obtenues en comparant les annotations de 2 annotateurs (A1 et A2) pour un texte donné. Si l'on considère A1 comme annotateur de référence, le rappel est le nombre d'annotations correctes trouvées par A2 parmi l'ensemble des annotations de référence, la précision est le nombre d'annotations correctes de A2 parmi l'ensemble de ses propres annotations. La formule pour calculer la F-mesure est : fsc $=(2 \times$ rec $\times$ pre $) /$ (rec + pre). Le résultat obtenu est compris entre o et $1: 1$ signifiant l'accord parfait, o le désaccord total. Il est traduit en pourcentage dans nos tableaux.

29 Ainsi act $=$ cor + par + inc, pos $=$ cor + par + inc + mis, pre $=$ cor $/$ act et rec $=$ cor $/$ pos (calculs et abréviations sont expliqués dans ce document en ligne : https://www.aclweb.org/anthology/M98-1024.pdf).

30 Une quatrième valeur, ERROR, a été ajoutée pour rendre compte des cas, extrêmement peu nombreux (il y en a quatre au total), où l'une des annotatrices a désigné comme entité spatiale une expression qui ne l'est manifestement pas.

31 Ce taux est calculé en divisant le nombre de cases vides dans la colonne "Difference " (donc des entités dont à la fois les bornes et les étiquettes coïncident) par le nombre total d'annotations « alignées ", qui est de 601 dans le cas des 5 échantillons retenus pour cette partie de l'analyse. La population observée étant plus importante, puisque à la fois les annotations de A manquant chez $\mathrm{B}$, et vice-versa, sont étiquetées, il est normal que le taux « moyen » soit plus bas que lors de l'observation de la F-mesure.

32 Toutes les citations de ce paragraphe sont extraites de l'échantillon Aldo si Aminta (roman de Constantin Boerescu, paru à Bucarest en 1855), dans lequel on constate le plus grand nombre de divergences impliquant "topical_space " d'une part, et " place " de l'autre. Les graphies d'origine ont été respectées, elles correspondent à un état de langue non stabilisé, marqué par le tout récent passage à l'écriture du roumain avec l'alphabet latin. Les entités concernées par l'annotation sont en italiques dans la traduction. 


\section{Auteurs}

\section{Ioana Galleron}

UMR 8094 Lattice, université Sorbonne-Nouvelle, Paris, France

Professeur de littérature française et des humanités numériques, Ioana Galleron est spécialiste de l'analyse littéraire assistée par ordinateur.

ORCID 0000-0003-0393-4485

ioana.galleron@sorbonne-nouvelle.fr

\section{Roxana Patraș}

Institut de recherche interdisciplinaire, université Alexandru Ioan Cuza, Iași, Roumanie Roxana Patraș est une chercheuse spécialisée dans l'histoire du roman roumain populaire, la littérature et la rhétorique politique à l'époque moderne.

ORCID 0000-0003-2534-8663

roxana.patras@uaic.ro

\section{Camelia Grădinaru}

Institut de recherche interdisciplinaire, université Alexandru Ioan Cuza, Iași, Roumanie Camelia Grădinaru est une chercheuse spécialisée dans l'étude des nouveaux médias. ORCID 0000-0002-3019-3056

camelia.gradinaru@uaic.ro

\section{Frédérique Mélanie-Becquet}

UMR 8094 Lattice, CNRS, Paris, France

Frédérique Mélanie-Becquet est ingénieure d'études en production, traitement et analyse de données, ayant participé à l'élaboration de différents corpus. Elle a plus particulièrement en charge l'élaboration de bases de données linguistiques.

frederique.melanie@ens.fr

\section{Droits d'auteur}

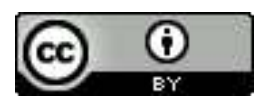

Les contenus de la revue Humanités numériques sont mis à disposition selon les termes de la Licence Creative Commons Attribution 4.0 International. 